\title{
CONSERVATION 4.0. POSSIBLE GUIDLINES FOR STANDARDISING THE DOCUMENTAL PROCESS FOR ARTISTIC HERITAGE.
}

\author{
F. Gasparetto*; L. Baratin* \\ * Conservation and Restoration school, DiSPeA, Piazza della Repubblica, 13, University of Urbino PU \\ francesca.gasparetto@uniurb.it ; laura.baratin@uniurb.it
}

KEY WORDS: conservation, standard process, data recording, data managing, data representation

\begin{abstract}
:
The contribution addresses the definition of a new standardised method for the documentation of the restoration process. In the frame of a conservation laboratory open to the public, the research drew up the guidelines for a documental and representative methods discussing the definition of a contemporary conservation praxis facing with digital technologies.

The research stems from a restoration of a Polyptych, Madonna con Bambino, Santi e Apostoli by Vittore Crivelli, damaged by earthquake of 2016 and temporarily showcased at Pinacoteca of San Domenico in Fano (PU). The public could visit the workplace set up and use the experimental informative system created from conservative data and results of intervention. To do this, the general process of documentation has progressed in strong synergy with restoration work. Thanks to the collaboration between experts, the project has established a set of priorities in order to capitalise the global results and better communicate each phase of intervention. The content of the study focuses on identifying guidelines that would form a possible framework for documentation useful for conservation of artistic heritage.

The standard workflow presented in the paper may potentially influence the theory of conservation as well as the practice. Developing a new approach to heritage, the study aims at bringing digital strategies into the field of conservation in order to propose the new idea of Conservation 4.0.
\end{abstract}

\section{INTRODUCTION}

Over the last ten years conservators increasingly find themselves asked to talk about their work and to explain the results of their conservative efforts. Lectures, panels, and sometimes special exhibits have accompanied the general public behind the scenes, inside the laboratory, in order to narrate a silent profession. Some famous examples of the last years are the restoration of the American Star-Spangled Banner in the National Museum of American History in Washington (Thomassen-Krauss, 2013), the restoration of The atelier du painter of Courbet in Museé d'Orsay in Paris, the restoration of the Nightwatch of Rembrandt in Rijksmuseum of Amsterdam or the intervention on the Leonardo's wall paintings at Castello Sforzesco in Milan.

In particular, these activities have been always viewed as an advertiser and funding tool, because they have the potential to define in depth why and how much cost a correct intervention of restoration. But they could be also interpretated as an important strategy to involve communities in the processes of heritage care to create social capital.

Starting from this observation, the research outlines an innovative field for conservators trying to interface the profession with the digital evolution and with the possibility of sharing that the internet offers today.

The contemporary inclination of museums and cultural institution to open up the internal processes as a participatory tool is directly connected to what we can call "digital think", which has expanded the borders of information and radically changed our models of knowledge.

In this context, in which conservation could be the "hot topic" of a different way for heritage outreach, we need to study new approaches for documentation and for following dissemination that, in particular, the innovative ICTs provide for easily using the rich cultural content of the field.

The paper provides an overview of strategies and technologies today applied in the field of artistic heritage conservation and identifies good practices for sustainable evolution of conservation activities in our digital world.
The study developed in two different and organic steps. The first necessary approach was the organisation of a scientific review in order to investigate the technical and technological state of the art. Describing the contemporary tools for digital analysis and documentation of artistic heritage, a catalogue was completed as a specialistic guide for professionals.

Moreover, the first section focuses on an extensive review of the literature about what is today the digital cultural heritage addressed to artistic objects, also highlighting some empirical examples to better outline the state of the art involving operational aspects.

The second and more substantial phase looked at the definition of an innovative digital method for conservators using the results collected in the scientific catalogue. Data and content produced during a conservative intervention have many possibilities of analysis, management and use offered by digital tools and media. Recording physical characteristics, documenting the restoration phases, organising sets of historical information could be a way by which the public apprehend the importance of an object, using all data produced by a conservative intervention.

But, as indicated by Santana Quintero (Santana Quintero et al. 2019), to correctly use these contents, we need standard and ethical principles guiding our documental work.

Following this principle and answering to the communication need of conservation sector declared for almost twenty years, the research has experimented with a documentary standardised method using digital technologies.

The pilot study was carried out in an restoration laboratory open to the public. The project named Open Restoration was a great opportunity to sketch theoretical suggestions and apply practical indications. Also, the suggested digital solutions improved the practice of conservators were working in the laboratory, sharing and preserving records among involved professionals.

The contribution seeks to establish a framework to review and apply a conservative method to improve the field of digital heritage recording. Moreover, this article arises from an interest in the way we communicate conservative information and the way our communication is changing as digital recording techniques. 
In this context, the research unfolds in the area of drawing and representation and investigates restoration processes focusing on the flow of digital documentation. The main goal is to outline theoretical and practical ways that get closer to contemporary thinking, which are taking in to account the participation of nonexperts as well.

The idea behind the research path has been to design a conservative procedure that considers the documentation phase as a basis for collaborative processes, being a useful tool for transmitting the importance of conservation and restoration for our society and culture. A proposal for current conservator practice was suggested, examining opportunities and challenges offered by the use of technologies and defining a new role for conservator of tomorrow.

\section{HOW DOCUMENT A CONSERVATIVE PROCESS}

\subsection{The state of the art}

The use of inventories in the documentation of cultural heritage is based on current best practices and on different practical experiences (Apollonio et al, 2019; Baratin et al., 2019; Bianchini et al., 2019) Over the years researchers and scholars always emphasised the importance of setting technical standards to dialog to different categories of experts and correctly transfer the cultural legacy, in particular the one of artistic objects.

More recently many charters and European documents tried to pinpoint the concept of "integrated conservation", incorporating the conservation of the heritage as a fundamental component of regional and cultural planning and the documentation as a principal need to start to protection path even more collaborative (Gustafsson, 2019).

Recording has become one of the key activities in conservation of cultural heritage. Producing adequate records of our actions, be it research, investigation, or treatment, not only is an ethical obligation for posterity but also implies immediate benefits in terms of project planning, interdisciplinary communication, and evaluation of results (Letellier, 2007).

In brief, the goal was to deeply analyse and register objects and sites in order to achieve a global knowledge sharable with the widest possible public.

The technical data collection of today is the documentation that we will use tomorrow to communicate with an unintended audience as well. And that is not the audiences the creators or specialists (primarily other conservators, or in some cases architects), but audiences who are examining knowledge models and graphical representations that were not built specifically to respond to their needs or questions (Rabinowitz, 2019).

In order to reach harmonisation between all kind of data, coordination and the adoption of standards are necessary as well as to implement an easy participation. Indeed, consideration and development of standards are important for several practical reasons, even more so today facing with digital applications (Ioannides et al., 2005).

The work started with the definition of some crucial key words allowing as to draw the best possible picture of the state of the art to which we were interfacing and from which we were starting. To help us in proposing a common and useful vocabulary for the field of art heritage documentation, three words were chosen during the study to define the documentation process. Collect, manage and represent are the three steps of a recognised documentation process.

\subsection{Collecting}

Our research trip started from the necessity to carry out recording to common standards and levels appropriate to the significance of the movable artistic heritage, as a fundamental activity in the conservation life of that heritage. A correct starting knowledge, in this sense, is absolutely essential.

This assumption goes on to suggest the interest in analysis of geometric and morphological data registration as the first approach to recording process.

A study of existing data collection methods and techniques was framed focusing on the current digital workflow for 3D models. Numbers of scientific studies analyse the modern digital technologies developed in cultural heritage sector (Pamart et al., 2019; Patrucco et al., 2019; Stathopoulou et al., 2019; Remondino et al., 2017; Sicuranza, 2013). By the study of these applications presented in various congresses and papers, a current scientific framework was sketched.

Today the methodological and technical approach of the Reverse Engineering (RE) is currently applied to movable cultural heritage thanks to its attention to detail and its accuracy in describing the metric data, that can easily be adapted to cultural objects of different shapes and dimensions. RE applies the analytical process of object 'deconstruction': starting with a highresolution survey of the parts, the object is virtually disassembled, and its design and operating mechanism are described.

The potential of these applications makes it possible to choose the instrumentation to be used according to the characteristics of the object or the information to be documented.

In the last twenty years, the use of these instruments in the field of cultural heritage has largely increased, making it almost an established practice in the case of major restoration or conservation studies.

In the particular case of the survey of movable heritage, the techniques used to build a path in the Digital Heritage field, according to the contemporary meaning of the term (Baratin and Gasparetto, 2020), can be grouped into two families: range-based and image-based acquisition techniques.

\subsection{Managing}

In the documentative pipeline, the second step is the management of all kinds of data collected. The imperative to manage intelligently accurate information has long been acknowledged by the cultural heritage community. Indeed, information needs to be preserved in order to be used by actual and future generations. But, despite the requirement, the management activities have been often difficult to implement.

The experiences analysed in the museums and cultural institutions around the world suggest that existing management systems are not properly systematised. In particular, in the field of conservation of movable heritage, the studies highlight a reluctance in creating a standard procedure.

The conservation schedule should contain many different types of data. In fact, it requires a complex structure that must provide for the possibility of compilation by different users and space for the various languages involved. The difficulty in defining a standard protocol, both in terms of the data to be entered and the architecture of the card itself, is certainly due to the heterogeneity of the data to be stored.

In the Italian context, the SICaR software moved close by the conservative data management approach. Thanks to an experimental process started in 20051, SICaR has developed a technical tool for geo-referenced documentation of the

\footnotetext{
${ }^{1}$ http://www.artpast.iccd.beniculturali.it/index.php?id=10
} 
restoration site, from the design phase to the execution phase (Baracchini, 2007; Siotto et al., 2016).

On the other hand, a well-designed cataloguing method was developed by ICCD (Istituto Centrale per il Catalogo e la Documentazione, Italian Ministry of Culture).

However, it is important to recognise the substantial difference between a conservation record and a catalogue record, because the approach to data collection and organisation is different. If, on the one hand, cataloguing produces alphanumeric and photographic data collected and organised in a formal and standard manner, the description of a work from a conservation point of view requires the participation of several professionals and the production of different data, with the consequent difficulty of defining a unitary approach: from three-dimensional digital models, to the graphs of the diagnostic analyses, to the historical information of the previous interventions and others. It is therefore formally different the figure who can work on the compilation of one or another sheet.

The ministerial organisation has developed a structure for the data entered in the catalogue card according to a system organised in paragraphs, defined by simple or structured fields (which in turn can be subdivided into subfields), which has made it possible to define a common national format for collecting alphanumeric cataloguing data: the catalogue card. The catalogue cards are schematic models organised to collect data (descriptive, technical-scientific, geographical, administrative) and to control the flow of their acquisition; every piece of data entered must follow the principles of the national standards, which regulate cataloguing activities for the heritage. Today, all the data and information collected is organised in a computer system, SIGECweb $^{2}$, an online platform that manages the entire flow of cataloguing.

This is a first demonstration of how the development and strengthening of an increasingly accessible way of understanding data management and use, thanks to a daily use of digital technologies and the web, is leading to a change in the configuration of the structure of databases.

\subsection{Representing}

In the documentative process, the word representing means communicating and it is the last step.

By profiling one or more possible outputs of the collected data, all useful forms are found to be understood and used through a common language. In fact, the aim is primarily to identify an uniform grammar that governs the use of words, images and graphic design, technological tools, and their results. It is therefore important to define languages, tools and channels of representation and communication.

The current state of the art is confused. It was hard handling the information and to coordinate the outcomes obtained from contemporary ICTs. Indeed, new technologies led to important technical results and to a following evolution of the concept of representation, today always more linked with perception (languages), with the use of devices moderators of interaction with the products of documentation (tools), with the accessibility of an heritage 'at your fingertips' used through the web (channels).

The study has organised this section analysing different practical experiences: from the investigation about the digital libraries to the survey on different applications used to visit museums and to focus on artworks; from dedicated website for the digital heritage

\footnotetext{
${ }^{2}$ http://www.catalogo.beniculturali.it/sigecSSU_FE/Home.action?timest amp $=1521647516354$

${ }^{3}$ www.openheritage3D.org
}

fruition $^{3}$ to scientific projects dedicated to opensource use (Potenziani et al., 2015).

Gaining knowledge, a general frame was sketched. In conclusion, it is possible to affirm that research is moving towards an increasingly broad digitisation of documenting practices for movable cultural heritage such as paintings, sculptures, and contemporary works of art. The process of opening up the use of digital techniques and technologies to an audience of restorers and conservators is ongoing thanks to collaborations between research centres, museum institutions and universities.

\section{OPENING UP THE CONSERVATIVE PROCESS}

\subsection{The innovative challenge for documentation of artistic heritage in the era of digital heritage}

To define the operational framework, it was necessary to study and examine in depth the topic of digital cultural heritage. Considering the ongoing debate about the professionals who are involved in this digital transition of cultural heritage, the analysis defined some knowledge gaps. It is important to note that this sector is currently under development and for this reason it was necessary to analyse some theoretical definitions.

Through this information, I developed a timeline starting from the first conceptualization of virtual heritage dating back to 1999 (Stone, 1999). Then trying to connect the various European documents, charters and projects about this topic a network of digital cultural heritage was created.

In particular, describing the theoretical evolution of the topic and underlining the increasingly more important role of documentation, some practical applications are evaluated, and it was clear the general effort to make the heritage even more accessible and open. One above all, the Europeana project - for nearly 15 years - has been committed to support the cultural heritage sector in its digital transformation, figuring out that it could be powered by digital culture.

The numbers of activities presented over the last years to open up the conservative process were the sign of a slow transformation of sensibility for heritage care. Reference is to many congresses (IIC, Conservation and Access - 2008; ICCROM - 2013) or special exhibitions and dedicated publications ${ }^{4}$. Likewise, the implicated digital technologies are an evident transformation in language, society and culture engaging simultaneously with virtual tools, web interconnectivity, and all multifarious applications. Digital technologies have become very important in our lives and the conservation sector could take advantages to answer to its needs and to face new challenges, that have not yet been fully imagined or critically explored.

These small transformations could lead to an interesting and radical change. Recognising a scientific role for communicative applications that talk about restoration and about how to take care of our heritage would lead to important results from a cultural point of view, because it would shorten - or even cancel - the gap that normally exists between a general visitor and the subject of restoration and conservation.

We should now focus on not just practical and singular experiences, but also on definition of a scientific sector with its ethical implications and theoretical considerations. If we don't, we risk missing an opportunity for growth and protect our heritage in many innovative ways.

\footnotetext{
4 http://www.vam.ac.uk/content/journals/conservation-journal/issue41/the-importance-of-being-less-earnest-communicatingconservation/
} 
This means that it is important that the experimental practices allow for better planning a standard digital workflow for the conservation of artistic objects. We need to apply a digital approach, organising all data from a conservative intervention in a pro-active network.

The research goes into the core of the presented issue investigating how to structure a conservative process that enables its communication using cutting-edge technologies.

The study started from the reinterpretation of the state of the art adapting some new solutions and proposals for the conservation context such as a laboratory. Practical experience has led to the definition of the general criteria, which design a method starting from the three keywords of heritage documentation: collect, manage, represent.

A new knowledge model supports the conservator in the phases of the traditional documentation method thanks to a conscious use of digital tools.

\section{A PILOT EXPERIENCE: THE OPEN RESTORATION PROJECT}

Up to this point the study presented has defined an integrated framework, where digital technologies improve the field of conservation.

In order to guarantee an accurate knowledge-system, the research has investigated the process of documentation typically used inside a laboratory and, starting from the observed results, the research has designed a possible digital method to collect, to manage and to represent the data.

The principal case study of the research is the project named Open Restoration, a pilot experience to test the innovative documentative process.

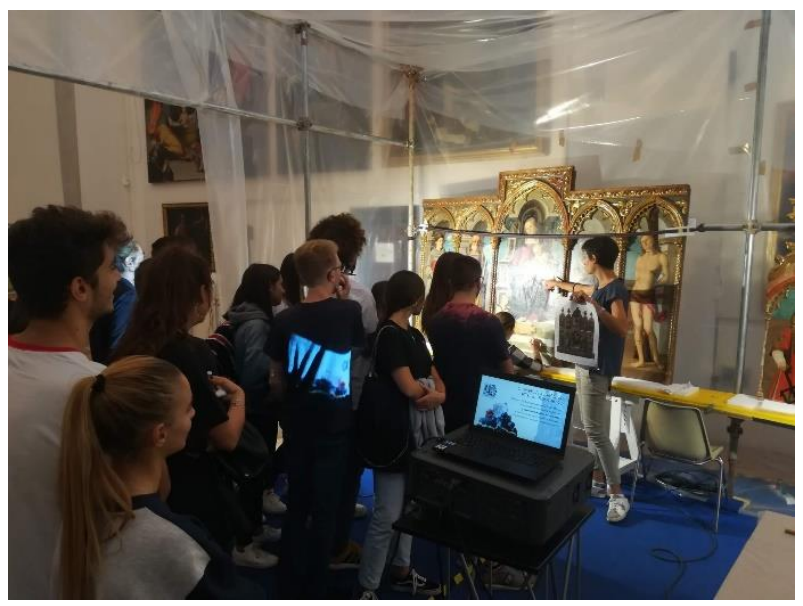

Figure 1. Conservator with visitors.

From January 2019 until October 2019, a Polyptych was conserved on public view in Fano, an Italian seaside village in the Marche region. A video, some supporting panels and a website totally focused on the documentation process supported the communication efforts.

The Open Restoration project was a real open laboratory. No glass walls were set up, promoting the pro-active visitor's participation.

The ambition of the project was to identify a method by which the technical data collected during the intervention could create a sort of a digital gallery, displaying in real time the operations carried out. From conservator's vantage point, we tried to translate the information about a specialist profession for a diversified audience.

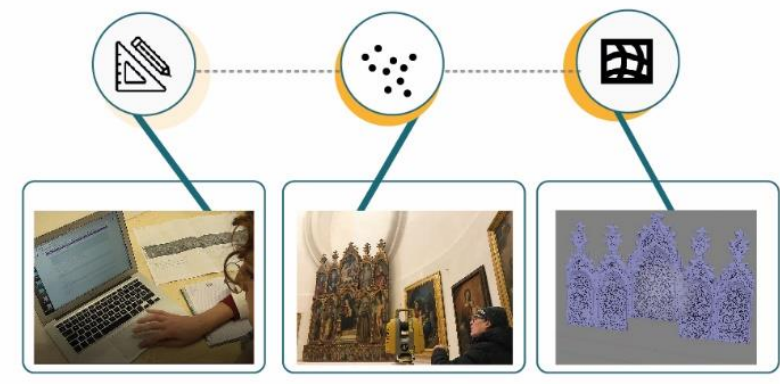

Figure 2. Pipeline 'KNOWLEDGE' phase

The Pinacoteca of Fano is a small exhibition space with no rigid exhibition paths. Initially, we set up the laboratory creating an accessible area where treatments could easily be viewed. But soon we realised without using ICTs we were missing an opportunity to better explain the conservative process.

In fact, as the project progressed, it was necessary to organise the amount of data and the following generated knowledge. A new application was added, sharing where the research had taken to conservators and the resulting new scientific identifications.

The visiting public wanted personal contact and for this reason we gave them the possibility to explore the results of intervention with the same technical tool used by conservators. The documentation was planned to be an open network, accessible to visitors and technical staff at the same time and with same channel.

For these reasons, replicable operating protocols were designed in order to collect and organise information.

Referring to three keywords mentioned above (collect, manage and represent), the study analysed the documentation flow composed of three phases: knowledge, management, and representation.

\subsection{Creating knowledge}

The principal goal of the knowledge phase was the collection of all data about the object and about its state of conservation.

What we can call 'informed conservation' is possible only with recording all data. Recording activities are intended as a prime responsibility of everybody involved in conservation processes. In fact, all those involved with the heritage care must have access to existing information and will generate records, which must be preserved and made available to others.

But the tasks of heritage conservators and restorers are not so clear and the policies and programs for a correct recording process are not jet so evident.

In the lack of prescriptions and codified indications regarding what should be the first approach to the realization of a correct documentation of conservation processes for movable heritage, in our research the constant reference is represented by the practical approach of the architecture sector. Therefore, the first step necessarily looks at the survey practices and at the morphological description of the object in order to define a documentation method.

We know that the survey techniques are considered as a set of preliminary operations aimed at knowing the dimensional data, the geometric configuration, and the description of materials of the object. These are considered as essential activities of all phases of the conservation process of buildings or sites and should be fully integrated into the general process.

So, involving these operational skills in the conservative process for artistic artworks, we were able to complete the object description, to record the dimensions (general and particular) and 
to visually describe its structural articulation, the surface characteristics, and its state of conservation.

For these reasons, digital metric surveys should represent the conservator's first approach to design a correct intervention.

The operational pipeline of this phase is composed of three steps: process design, data acquisition, processing of the acquired data. By obtaining datasets of different digital information (from HD photographs to the 3D model), a virtual model of the real object is the principal result, with different precision levels of aesthetic reproduction that depend on the techniques used.

The first digital product allows the morphological investigations of the structure and the studies about the state of conservation of materials.

As is evident, a new expressive digital language is obtained from this first step, transforming the physicality of the object. The innovative language is flexible and adaptable to the medium of use. This is the transformation from 'matter to be preserved' into 'material for study and research to be transmitted'.

\subsection{Managing the process}

The management constituted the second step of the process, and it was the core of the study. It aimed to design a flexible digital repository.

The rapid rise in new digital technologies has revolutionized the practice of manging data for heritage objects. Digital tools and media today offer a myriad of new opportunities for collecting and using information. But with these new opportunities, there are also many problems about the appropriate level and degree of managing and documentation knowledge required by conservation professionals. Up to now no prescriptions and no indications exist about a general and standard flow in organising information.

In this context, the study analysed different typologies of data created within the laboratory. Indeed in a conservative work we produce different typologies of information. As Rabinowitz already said, for example, we can face with paradata or with metadata. Paradata are differentiated from the metadata that describes a digital object or its subject (Rabinowitz, 2019). In fact, according with the explication of London Charter, paradata are "information about human processes of understanding and interpretation of data objects".

Starting from this consideration, our managing process should take care of different levels and typologies of information, in order to explore a critical approach to the development of a standard process.

The repository project has involved a sort of card catalogue arranged in a chronological order that documents the evolution of the conservation intervention. Tipology-level documentation of conservation process was designed, in order to create a feasible online collection form of technical data, considering changes required for different objects in term of documental procedures. Consequently, a flexible ontology of the digital schedule was obtained and a possible architecture for the open digital repository was designed.

During the phase of organising the collected data, it is necessary to use a technology able to interconnect several users, in order to maximise the integration of the information and make it shareable.

The result was the creation of a digital inventory of conservation phases that differed regarding the motivation of conservation, typology of object, accessibility for different users, and content. Activities particularities, differences in the technical descriptions of object and in competences of involved professionals influenced physical, informational and procedural scopes of the registration process.
The impact of data management on the entire document process is high, since this phase is a key moment, i.e. the step in which the information collected is put into a system and then made usable.

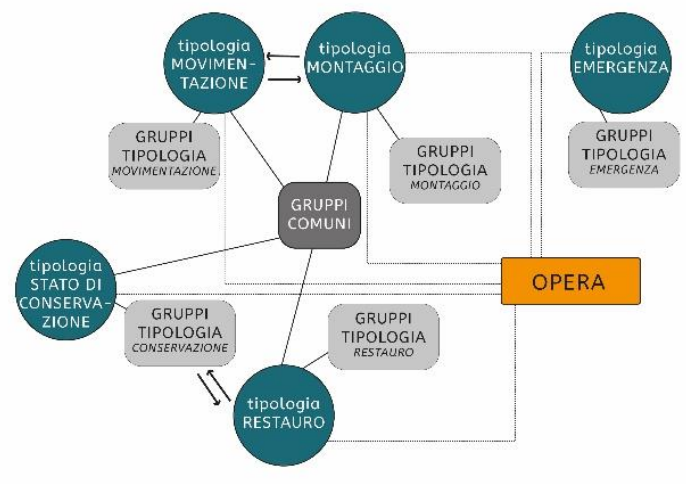

Figure 3. Schematic ontology of the conservative schedule

The challenge was to create a new managing model for online documentation able to organise technical aspects of conservation into effective and sustainable knowledge. The potential of technology liberated documentation from an empirical model and it allowed in reaching a standardised description to incorporate different narrative levels.

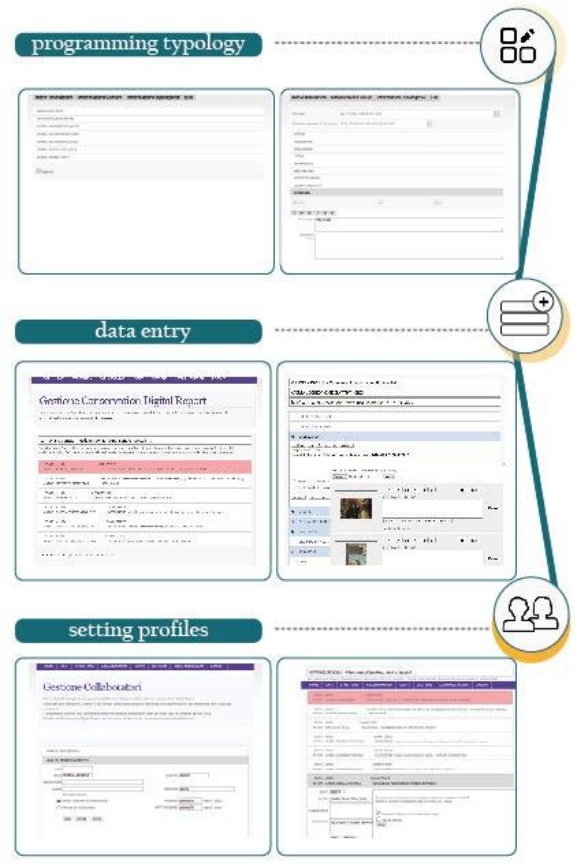

Figure 4. The flow of management

So, what are the implications for objects conservative documentation? First, we needed to program the digital 'working-typology'. In the case of Polyptych, we designed the structure of the digital schedule based on technical and morphological characteristics of the artwork: its complex structure composed of three independent parts, its wooden support, its state of conservation and so on.

Digital technologies and digital information systems offer the possibility to customise the final outputs, with the advantage to emancipate the description from a rigid documentative structure. Then we started the data entry. Photos, links and general information are inserted in the web repository. 
Finally, to provide an open structure, different profile are activated enabling the inclusion of relevant professionals and sharing paradigm.

\subsection{Communicating the results}

The last phase, the one about representation, aimed at two goals: one generic addressed to visitors and a second technical for professionals. The first one is about the possibility to define and then test a new language for open documentation, shareable with the widest possible public; the second is to study and to design a possible graphic standard to outline the graphic representation of the state of conservation, artistic techniques or interventions. In any case, the two goals are closely linked by the need to communicate the collected data we just processed and organised into the repository.

Principal aim of this phase is to reflect on the communication challenges of digital heritage obtained from a deep documentation work and it would compare digital contents to real contents and their tangible reference. Highlighting the role of visual perception and comprehension of mapping activities, this section of the study focuses on the nature and characteristics of digital representations, intended as complex data-systems.

The Charter on the Preservation of the Digital Heritage ratifies the importance of digital tools and methods in the creation of cultural heritage (UNESCO, 2003). In a same way, the London Charter focuses on the possibilities to correctly communicate the heritage through digital language.

Referring to the cited documents, the study identifies and proposes a possible standard to obtain a common language for professionals and non-experts.

The study started from the research of a graphic standard. A graphic standard is effectively a set of visual regulations for the guarantee of the correct communication and protection of the long-term value of digital data for the storage, exchange, sharing, searching, visualizing/presenting and retrieval of information between different users/professionals (Ioannidies, 2005). The standards are our guidelines in representation, and they can help all experts involved in the cultural heritage area in the visual organisation of data.

The first analysis focused on the selection of the types and thicknesses of the representation lines, the language that regulates the shape synthesis of the object.
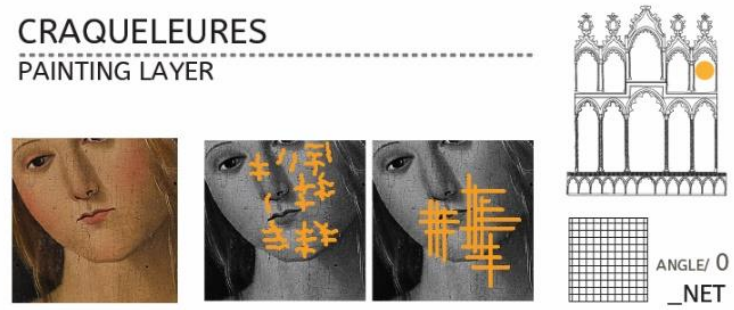

Figure 5. An example of standard for the representation of a type of damage on painting layers

Centring the research on summary and figurative graphic representation, we studied a visual solution able to communicate all peculiar aspects of the artwork. In contrast with architecture and design objects, paintings and artistic sculptures are characterised by figurative aspects not to be underestimated. For this reason, we decided to support the elaborated outputs of the survey with a black and white orthophoto in transparency, to help the comprehension of the pictorial surface.

Finally, a graphic standard has been studied on decay of paintings layers and wooden support, and for the synthesis of the set of interventions. Starting from a detailed photo of each degradation, a simplified and graphitised solution was developed.

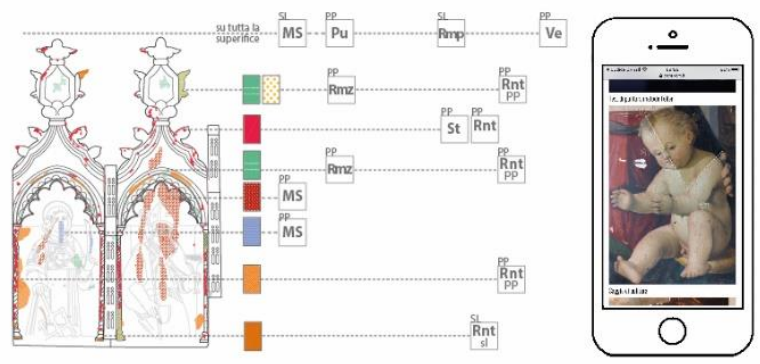

Figure 6. Synthesis of proposal of standard for technical and general communication of interventions

The study pursued other objectives. In particular, the experimentation wanted to involve the public in the evolution of the conservative work. The new audience consists of the general public, which is for the most part not interested in deriving scientific information, but they are primarily interested in the opportunity to explore different phases of intervention in an easy way. They might use the proposed models and maps to understand the result of an intervention and to perceive its value, also asking new questions, as well as a growing public community becoming more aware.

So, the second approach to the theme 'documenting for communicating' therefore exploits the technical tool with which the data was managed: the digital web schedule.

From a certain point of view, it approached the development of an open data model: the open data of the Open Restoration project made available to technicians and non-experts as well. In this context, communication has to be managed at several levels, from the expert to the general public, through the use of calibrated representative tools that facilitate access to knowledge. Norms and standardisations regulating a scientific translation of the results composed a sort of general guideline for conservators. These guidelines must necessarily refer to a new grammar of representation and communication.

Therefore, starting from a technical glossary of reference, which should be followed like a grammatical code since it becomes the common interpretation tool for all users, the step of representation can be understood as that of translation. Graphic representation and design play the fundamental role of informing, with the awareness that visual language is able to disseminate content quickly, correctly and effectively.

\section{CONSERVATION 4.0}

At the end of the work on Polyptych, two results were presented. A technical booklet, with all information regarding materials, degradations, and details about the intervention. In this case, a specific graphic standard was studied in order to propose a new possible language for professionals. In the context of conservation, graphic language has never been systematised before. That is why we looked at the method used in architectural approach in order to adapt those rules to the field of movable assets.

The second result was addressed to the public. We already said that during the restoration in the Pinacoteca, visitors could observe restorers at work and, moreover, thanks the digital experimentation they could investigate the results via smartphone by framing a QRcode.

Once intervention was terminated, the painting was displayed in an exhibition in Rome. Here, a set of panels and a video were carried out to talk about the aspects of Open Restoration project. 
In that situation, through the use of ICTs, it was possible to share the whole experience of the open laboratory even after the work had been completed, with the aim of promoting the project and the conservation of the artwork in an increasingly proactive way. From the example of Open Restoration project, we tried to design a possible methodology, a sort of guideline to help the restorer during his/her work to create a well-done documentation. Three criteria result from the research and concern different aspects of documenting the process.

The first is about digitalisation, the transformation of materiality of the object into digital content. The preliminary step has to do with the digital collection of data, starting from a digital survey. The second criterion talks about a sharing paradigm and the ability of digital tools to manage different data and documental levels. Finally, the third criterion is about the representation. Representation is the final step of the complex process of documentation, and it aims to communicate with the public. Of course, as guidelines they are just suggestions, but they can be adopted according to the actual need to digitise the field of restoration, in order to create an interaction with a contemporary evolution. In fact following guidelines is never mandatory, but they can be an operative actualisation of theoretical expertise. This study must be considered as a starting point in the exploration of the concept of Conservation 4.0, that represents the complexity of the digital experience for the restoration and conservation field, in order to accept the digital evolution.

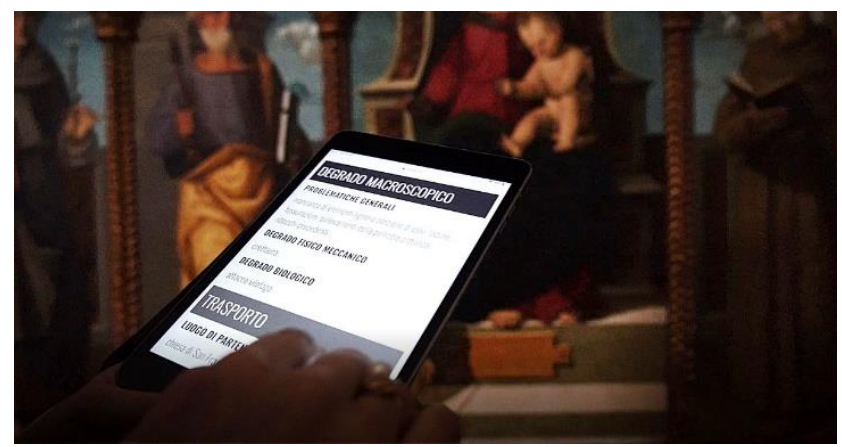

Figure 7. The use of digital schedule during the regular activities of monitoring.

So, what does 'conservation 4.0 ' means in concrete terms? Imagining a new way of documenting the work of restorer, 'conservation 4.0' can be defined as an experimental paradigm which supports the laboratory's regular activities. The importance of the conservation is communicated as a synonym of care of the cultural heritage involving the citizens. The idea of an open laboratory is translated into a narrative approach, as inclusive as possible. In the case of Polyptych, for example, a technical documentation software has been transformed in a communication tool and virtual places open to the public.

\section{FUTURE PERSPECTIVES AND POSSIBLE CURRENT APPLICATIONS}

The content of this paper focuses on identifying guidelines that would form a framework of applicative principles for conservators and restorers involved in the documentative process as a real part of an intervention. In fact, one of the key goals of the framework is to involve conservators in the communicative final step ensuring that the records produced are shared in the present and in the future using sustainable strategies.

The core of this research argues that emerging digital skills may benefit the rethinking of traditional restoration. In fact, by accepting this evolution it's possible to build a new contemporary profession with new perspectives that is even more sustainable in the future.

The innovative framework proposed by this study can open new possibilities and new opportunities to rethink the role of restorer in a digital panorama.

\section{ACKNOWLEDGEMENTS}

We wish to acknowledge the support of DICEA, Dept. of Civil and Building Engineering and Architecture, Polytechnic University of Marche of Ancona (Italy) and TOPCON Positioning Italy s.r.l. for technical support, Carifano Foundation for their hospitality inside the Pinacoteca of San Domenico, the Municipality of Monte San Pietrangeli original location of Polyptych. Finally, we wish to thank ANCI Marche, Pio Sodalizio dei Piceni and Artifex company for their financial support.

\section{REFERENCES}

(2003) Charter on the Preservation of Digital Heritage. Disponibile in http://portal.unesco.org/en/ev.php-

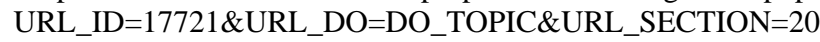
1.html [24/10/2020]

Baracchini, C., Lanari, P., Ponticelli, P., Parenti, R., Vecchi, A. 2005. SICaR: un sistema per la documentazione georeferenziata in rete. Scienza e beni culturali. XXI. 735-747.

Baratin, L, Scicolone, G., Lonati, S. 2016. Conservation Diglital Report: standard documentation in Cultural Heritage. In Catalano, C.F. \& De Luca, L (2016). EUROGRAPH Workshop on Graphics and Cultural Heritage. DOI:10.2312/gch.20161405.

Baratin, L., A.Cattaneo, F.Gasparetto, E.Moretti, S. Lonati. 2019. Documentig the conservative evolution of the city walls thanks to the integration of digital systems of various typologies. The case study of Valbona gate. The International Archives of the Photogrammetry, Remote Sensing and Spatial Information Sciences, Volume XLII-2/W11, 2019 GEORES 2019. 2nd International Conference of Geomatics and Restoration, 8-10 May 2019, Milan, Italy.

Baratin, L., Gasparetto, F. 2019. Contemporary restoration as an inclusive process. The new digital documentation challenges. In Disegnarecon, Experiential Design for Heritage and Environmental Representation. Vol.12, n.23.4

Baratin, L., Gasparetto, F. 2020. Digital Cultural Heritage: some notes from conservative experience. In Carmine Gambardella (edited by). World Heritage and Contamination. Abstracts of the XVIII International Forum 'World Heritage and Contamination', Gangemi Editor International Publishing, ISBN 978-88-4923936-2.

Bianchini, C., Casale, A., Empler, T., Esposito, D., Inglese, C., Ippoliti, E., et al. 2019. Ecosistemi Digitali e Risorse Culturali. Paesaggio Urbano. N.1. Disponibile in https://www.paesaggiourbano.org/2019/09/11/paesaggiourbano-2019_1/ [1 aprile 2019]. 40-51.

Bini, M. \& Bertocci, S. 2012. Manuale di rilievo architettonico e urbano. Roma: Città Studi Edizioni.

Gasparetto, F., L.Baratin, A.Devecchi. 2019. A multidisciplinary documental representation method for kinetic and environmental art. The International Archives of the Photogrammetry, Remote Sensing and Spatial Information Sciences, Volume XLII-2/W15, 2019 27th CIPA International Symposium "Documenting the past for a better future", 1-5 September 2019, Ávila, Spain. 
Gustafsson, C. 2019. Conservation 3.0 - Cultural Heritage as a driver for regional growth. SCIentific RESearch and Information Technology Ricerca Scientifica e Tecnologie dell'Informazione. Vol 9, Issue 1. ISSN 2239-4303, DOI 10.2423/i22394303v9n1p21. 21-32.

Ioannides, M., Georgopoulos, A., Scherer, M. 2005. Standards in cultural heritage: the missing grammar for the digital documentation of the past. Proceedings of CIPA $2005 X X$ International Symposium, Torino, Italy.

Letellier, R. 2007. Recording, Documentation and Information Management for the conservation of Heritage. Guiding Principles. Los Angeles: The Getty Conservation Institute.

Pamart, A., Ponchio, F., Abergel, V., Alaoui M'Darhri, A., Corsin, M. Et al. 2019. A complete framework operating spatially-oriented RTI in a 3D/2D cultural heritage documentation and analysis tool. The International Archives of the Photogrammetry, Remote Sensing and Spatial Information Sciences (Volume XLII-2/W9). Proceedings of 8th Intl. Workshop 3D-ARCH "3D Virtual Reconstruction and Visualization of Complex Architectures”. Italia, Bergamo.

Patrucco, F., G. Rinaudo, \& Spreafico, A. 2019. A new handheld scanner for $3 \mathrm{~d}$ survey of small artifacts: the Stonex F6. The international archives of the photogrammetry, remote sensing and spatial information sciences. - (Volume XLII-2/W15)2. Proceedings of 7th CIPA International Symposium "Documenting the past for a better future". Ávila, Spain. 895901.

Potenziani, M., Callieri, M., Dellepiane, M., Corsini, M., Ponchio, F., Scopigno, F. 2015. 3DHOP: 3DHeritage Online Presenter. Computer \& Graphic. N. 52. http://dx.doi.org/10.1016/j.cag.2015.07.001. 129-141.

Rabinowitz, A. 2019. Communicating in three dimensions: questions of audience and reuse in 3D excavation documentation practice. Studies in Digital Heritage, Vol. 3, No. 1.

Remondino, F., Nocerino, R., Toschi, I. \& Menna, F. 2017. A critical review of automated photogrammetric processing of large datasets. The ISPRS Annals of the Photogrammetry, Remote Sensing and Spatial Information Sciences (Volume IV-2/W2). Proceedings of 26th International CIPA Symposium 2017. Canada, Ottawa.

Sacco, F. 1993. Il problema della documentazione grafica dei restauri. Materiali e strutture. Problemi di conservazione. Anno III, numero 1. 25-34.

Santana Quintero, M. \& Vileikis, O. 2011. Heritage Recording and Infromation Management in Digital Age (SMARTdocheritage). Change over time. Vol. 1, N.2. 156-164.

Santana Quintero, M., Fai, S., Smith, L., Duer, A. \& Barazzetti, L. 2019. Ethical Framework for Heritage Recording Specialists Apply-ing Digital Workflows for Conservation. Int. Arch. Photogramm. Remote Sens. Spatial Inf. Sci. (XLII-2/W15). DOI: https://doi.org/10.5194/isprs-archives-XLII-2-W15-1063-2019. 1063-1070.

Sicuranza, F. 2013. Sperimentazione di sistemi di structure from motion per la restituzione di apparati decorativi. Carlevaris, L.(ed.) (2013). Linee di ricerca nell'area del Disegno. Contributi dalle tesi di dottorato in Mostra. Roma: Aracne Editrice.

Siotto,E., Baracchini, C., Santamaria, U., Scopigno, R. 2016. Sperimentazione del sistema ministeriale SICAR w/b per la gestione e la consultazione informatizzata dei dati sulla policromia. Archeologia e calcolatori. N.27. 131-151.
Stathopoulou, E.-K., Welponer1, M. \& Remondino, F. (2019). Open-source image-based 3d reconstruction pipelines: review, comparison and evaluation. The International Archives of the Photogrammetry, Remote Sensing and Spatial Information Sciences (Volume XLII-2/W17) Proceedings of 6th International Workshop LowCost 3D - Sensors, Algorithms, Applications. France, Strasbourg.

Stone, R.J. 1999. Virtual Heritage. UNESCO World Heritage Review. Oct. 1999, n.13. pp. 18-27.

Williams, E. 2013. The public face of conservation. London: Archetype Publications Ltd. 